\title{
Role of Cytokines Released During Pyroptosis in Non-Small Cell Lung Cancer
}

\author{
Yuanli Huang ${ }^{1,2}$ \\ Guanghui Zhang ${ }^{1,2}$ \\ Qing Zhu' \\ Xia $\mathrm{Wu} \mathbb{1}^{3}$ \\ Ligao $\mathrm{Wu}^{\prime}$
}

'Department of Pathology, the First Affiliated Hospital of Bengbu Medical College, Bengbu City, Anhui Province, People's Republic of China; ${ }^{2}$ Graduate School of Bengbu Medical College, Bengbu City, Anhui Province, People's Republic of China; ${ }^{3}$ Department of Pathology, Fuyang Tumor Hospital, Fuyang City, Anhui Province, People's Republic of China
Correspondence: Ligao Wu Tel +86 I5395225588

Email wlgahbb@I26.com
Purpose: Pyroptosis is a recently discovered highly inflammatory form of programmed cell death, during which the N-terminus of the cleaved Gasdermin protein family forms pores in the cell membrane, leading to cell disintegration and the release of certain intracellular factors, including caspase3, gasdermin E (GSDME), and high mobility group proteins (HMGB1), which trigger a series of secondary inflammatory reactions. Specifically, caspase3 can lyse GSDME and induce pyrolysis, while HMGB1 is released passively after cell membrane destruction. In this study, the roles of these proteins in lung cancer tissues as well as their clinical significance were investigated.

Patients and Methods: The expression levels of GSDME, caspase3, and HMGB1 proteins in lung cancer and paracancerous tissues were determined via immunohistochemical staining, and their relationship with the clinical stage, pathological grade, and survival prognosis of the patients was analyzed. Further, $\mathrm{CD}^{+} \mathrm{T}$ cell accumulation in the above-mentioned tissues was also determined, and differences between them with respect to $\mathrm{CD} 8^{+} \mathrm{T}$ cell distribution were also investigated. Furthermore, the relationships between $\mathrm{CD}^{+} \mathrm{T}$ cell abundance and the expression levels of the above-mentioned proteins were determined via statistical analyses.

Results: Lung cancer and paracancerous tissues showed significantly different GSDME, caspase3, and HMGB1 protein expression levels. GSDME expression level and the presence or absence of lymph node invasion were identified as prognostic indicators of survival in patients with lung cancer. Surprisingly, however, HMGB1, which showed a certain level of correlation with the presence or absence of lymph node metastasis, could not be used as a prognostic indicator of survival.

Conclusion: GSDME may be an important prognostic indicator of survival in patients with lung cancer. However, the effects of HMGB1 expression level and $\mathrm{CD} 8^{+} \mathrm{T}$ cell abundance on the prognosis of patients with lung cancer still need further investigation.

Keywords: cell pyroptosis, caspase3, GSDME, HMGB1, lung cancer, CD8 ${ }^{+} \mathrm{T}$

\section{Introduction}

It is well known that most cancer-related deaths are caused by tumor cell infiltration, invasion, and metastasis, rather than the tumor itself. ${ }^{1}$ Therefore, inducing or promoting tumor cell death and inhibiting their growth are the primary goals of cancer treatment. Further, for a long time now, it has been considered that the drug resistance and immune evasion characteristics of tumor cells are primarily responsible for tumor recurrence and treatment failure. Thus, the complex molecular mechanisms involved, which may provide new therapeutic insights for overcoming such challenges, need to be explored. Globally, lung cancer is the leading cause of cancer-related deaths, and 
when at the advanced stage, its 5-year overall survival rate (OS) is below $5 \%,{ }^{2,3}$ and current treatment options, with surgery being the primary strategy, are insufficient. We know that the majority of lung cancer patients with tissue credit type are non-small cell lung cancer, and the incidence and metastasis rate of non-small cell lung cancer are very high. Therefore, we used NSCLC as a disease entry point to explore the role of inflammatory factors released by gasdermin E (GSDME)-mediated scortosis and GSDME expression in the survival and prognosis of NSCLC patients. In order to find the internal molecular mechanism of tumor treatment, and open a new chapter of targeted therapy.

Pyroptosis, which is a recently discovered highly inflammatory form of programmed cell death, is characterized by cell apoptosis and necrosis, which trigger secondary inflammation. In 2017, Wang et $\mathrm{al}^{4}$ observed that gasdermin E (GSDME), a member of the Gasdermin protein family, can be cleaved by caspase3, resulting in the occurrence of pyroptosis, which begins with cell membrane rupture, followed by the collapse of the cell, and the subsequent release of intracellular factors. It has also been reported that cytokines play a key role in tumorigenesis and also participate in the interaction between inflammatory immune and non-immune cells and cancer cells. ${ }^{2,5}$

As a nuclear factor, high mobility group protein (HMGB1) is passively released after cell membrane destruction. Further, as a mobile chromatin binding protein, it can act as a DNA molecular chaperone, and when released into the extracellular environment, it can also function' as a pro-inflammatory cytokine. ${ }^{6,7}$ Generally, HMGB1 is passively released from dead, dying, or injured cells, which are not associated with apoptosis, ${ }^{8}$ and when in the extracellular environment, it is widely regarded as a signal molecule that participates in a variety of physical and pathological processes, triggering inflammatory responses, and activating innate and adaptive immunity via interaction with a variety of receptors. Further, HMGB1 released into the extracellular space recruits a large number of highly differentiated effector $T$ cells resulting in the accumulation of $\mathrm{CD} 8^{+} \mathrm{T}$ cells in the extracellular matrix during immune response promotion processes. Highly differentiated effector $\mathrm{T}$ cells have also been observed in several disease processes, including autoimmune diseases, organ transplantation, and cancer, ${ }^{10}$ and the immune surveillance ability of $\mathrm{CD}^{+} \mathrm{T}$ cells on tumor cells is conducive for the fight against cancer immune evasion strategies. Specifically, a study by Zhang et al ${ }^{11}$ indicated that $\mathrm{CD} 8^{+} \mathrm{T}$ cells mediate the tumor suppressor effect of GSDME, whose expression also enhances the number and function of $\mathrm{CD} 8^{+} \mathrm{T}$ lymphocytes.
Therefore, in this study, the clinical significance of the expression levels of GSDME, caspase3, and HMGB1 proteins in lung cancer tissues and the relationships between them were investigated. The distribution of $\mathrm{CD} 8^{+}$ $\mathrm{T}$ lymphocytes within lung cancer tissues as well as the relationship between the abundance of $\mathrm{CD} 8^{+}$ $\mathrm{T}$ lymphocytes and the expression levels of the abovementioned proteins were also explored.

\section{Materials and Methods Sample Collection}

In this study, based on diagnosis performed at the Pathology Department of the First Affiliated Hospital of Bengbu Medical College, 110 paraffin-embedded radical lung cancer resection specimens were collected from 110 patients with lung cancer between January 1, 2013 and December 30, 2014. None of the patients received radiotherapy or chemotherapy before surgery for sample collection. Further, the presence or absence of lymph nodes and distant metastasis of lung cancer, the survival status of the patients, as well as data on other parameters, were obtained from medical records and via telephone follow-up. Simultaneously, 20 paracancerous tissues were collected from 20 out of the 110 patients with lung cancer. All the experimental procedures, which complied with the ethical standards of the declaration of Helsinki, were approved by the ethics committee of Bengbu Medical College. [Renke Pi Zi [2021] No. 265].

Among the 110 patients, whose ages varied in the range 40-81 years (median $=65$ years), 85 were male and 25 were female. Based on clinical staging performed according to the American Joint Committee on Cancer (AJCC) Cancer Staging Manual (8th Edition), 35, 33, 40, and 2 of the 110 patients had stage I, stage II, stage III, and stage IV lung cancer, respectively. Additionally, among the 110 cases, there were 44 patients with lymph node invasion and 2 with distant metastases. All the patients were followed up until death or until January 1, 2020; the longest and shortest follow-up intervals were 84 and 60 months, respectively. Table 1 details the clinical characteristics of the 110 lung cancer tissue samples.

\section{Reagents}

Rabbit polyclonal antibodies against human caspase-3, HMGB1, and GSDME were purchased from Proteintech (Rosemont, IL, USA). Further, rabbit monoclonal antibody against human CD8 + T lymphocytes, the ElivisionTM Plus 
Table I Summary of Clinical Characteristics of I I 0 Lung Cancer Patients

\begin{tabular}{|c|c|c|}
\hline \multicolumn{2}{|l|}{ Characteristics } & \multirow{3}{*}{$\begin{array}{l}\text { Patient No. (\%) } \\
72(65.5) \\
38(34.5)\end{array}$} \\
\hline Tumor types & Squamous cell carcinomas & \\
\hline & Adenocarcinoma & \\
\hline \multirow[t]{4}{*}{ Age } & Range & $40-81$ \\
\hline & Median & 65 \\
\hline & $>65 y$ & $53(48.2)$ \\
\hline & $\leq 65 y$ & $57(5 \mathrm{I} .8)$ \\
\hline \multirow[t]{2}{*}{ Gender } & Male & $85(77.3)$ \\
\hline & Female & $25(22.7)$ \\
\hline \multirow[t]{3}{*}{ Pathologic grade } & I & $27(24.5)$ \\
\hline & II & $57(5 \mathrm{I} .8)$ \\
\hline & III & $26(23.6)$ \\
\hline \multirow[t]{4}{*}{ TNM stage } & Stage I & $35(31.8)$ \\
\hline & Stage II & $33(30.0)$ \\
\hline & Stage III & $40(36.4)$ \\
\hline & Stage IV & $2(1.8)$ \\
\hline \multirow[t]{2}{*}{ Lymph nodes } & Positive & $44(40.0)$ \\
\hline & Negative & $66(60.0)$ \\
\hline \multirow[t]{2}{*}{ Vital states } & Alive & $43(39.1)$ \\
\hline & Dead & $67(60.9)$ \\
\hline \multirow[t]{2}{*}{ Tumor size (Size of primary lesion) } & $\leq 5 \mathrm{~cm}$ & $82(74.5)$ \\
\hline & $>5 \mathrm{~cm}$ & $28(25.5)$ \\
\hline
\end{tabular}

Kit, and the DAB color developing kit were purchased from Fuzhou Maixin Biological Company (Fuzhou, China).

\section{Experiments}

At the Department of Pathology of the First Affiliated Hospital of Bengbu Medical College, serial sections ( $4 \mu \mathrm{m})$ were prepared on a formalin-fixed, paraffin-embedded tissue microarray. After the tissue slides were deparaffinized, hematoxylin/eosin and immunohistochemical staining were performed followed by histological observations under an optical microscope (Olympus Optical Company Ltd., Tokyo, Japan). Clinical staging was performed according to the American Joint Committee on Cancer (AJCC) Cancer Staging Manual (8th edition). Further, the immunohistochemical staining using the ElivisionTM Plus Kit was performed according to the manufacturer's instructions.

\section{Evaluation of Immunoreactivity}

The criteria for scoring GSDME expression level were as follows: The intensity was graded according to the following scale: 0 , negative; 1 , weak; 2 , moderate; and 3 , strong.
Thereafter, the proportion of positive tumor cells was graded as follows: $0,<5 \% ; 1,5-25 \% ; 2,26-50 \% ; 3$, $51-75 \% ; 4,>75 \%$. The final score was then computed by multiplying these two primary scores, and final scores in the range $0-6$ were defined as "low expression" or $(-)$, while those in the range 6-12 were defined as "high expression" or $(+){ }^{12}$ The same method was used to evaluate the staining signals for caspase 3 and HMGB1.

To determine the distribution of $\mathrm{CD} 8{ }^{+} \mathrm{T}$ lymphocytes, we listed the positive cells from the representative view of the three parts of the high power field (HPF, $\times 200$ magnification), and used the average value, with the critical value being 34 cells/HPF, ${ }^{13}$ which was determined using X-tile v.3.6.1 (Yale University, New Haven, CT, USA). Further, the immunohistochemical staining results were evaluated by two professional pathologists using independent double-blind methods.

\section{Statistical Analysis}

Statistical analyses were performed using SPSS version 25.0 (IBM, Chicago, IL, USA). The Kaplan-Meier method was 
used to analyze survival based on the expression levels of caspase-3, HMGB1, and GSDME proteins and the abundance of $\mathrm{CD}^{+} \mathrm{T}$ lymphocytes, and to draw univariate survival curves. Further, the Log rank test was used for comparison between groups, and the Cox multiple regression model was used for multivariate analysis to estimate the hazard ration (HR) and the $95 \%$ confidence interval (CI). The correlations between the expression levels of caspase-3, HMGB1, and GSDME in lung cancer tissues and clinicopathological parameters were analyzed using $\chi^{2}$ and Spearman rank correlation tests, while differences between lung cancer and paracancerous tissues with respect to the expression levels of caspase-3, HMGB1, and GSDME, and CD8 ${ }^{+} \mathrm{T}$ lymphocytes abundance were analyzed by performing the $t$-test. $\mathrm{P}<0.05$ were considered statistically significant.

\section{Results}

The Clinical Significance of the Protein Expression of GSDME, Caspase3, HMGBI and the Abundance of $\mathrm{CD} 8^{+} \mathrm{T}$ Lymphocytes in Patients with Lung Cancer

The expression levels of GSDME, caspase3, and HMGB1 proteins in lung cancer and paracancerous tissues were detected via immunohistochemistry. GSDME was found to be predominantly localized in the cytoplasm, and to a lesser extent, on the cell membrane, and its accumulation on the cell membrane perforated the cell membrane, resulting in the collapse of the cell and its subsequent death. HMGB1 and caspase 3 were found to be predominantly localized in the nucleus and cytoplasm, as shown in Figure 1. Further, the

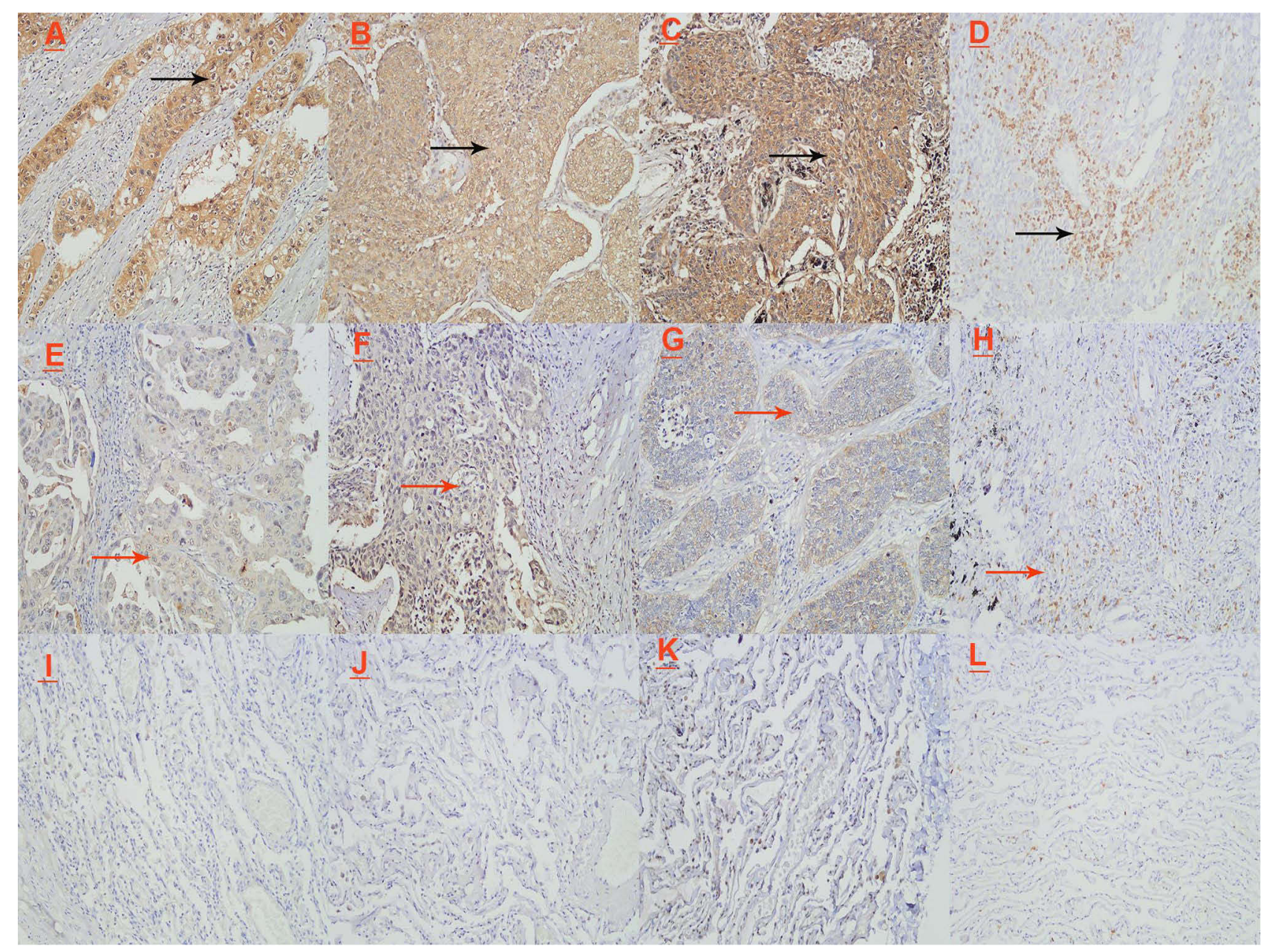

Figure I (A-C) represent the high expression of GSDME, caspase3 and HMGBI proteins in lung cancer tissues, respectively. (D) The expression of high abundance of CD8 + T lymphocytes in lung cancer tissues. (E-G) are GSDME, caspase3, HMGBI protein in lung cancer tissue low expression. (H) Low abundance of CD8+T lymphocytes in lung cancer tissues. (I-L) is the distribution of GSDME, caspase3, HMGBI, and CD8+T lymphocytes in paracancerous tissues. In the figure, the black arrows is a high expression image and the red arrows is a low expression image. (Images were acquired at $200 \times$ magnification.). 
positive expression levels of GSDME, caspase3, and HMGB1 and $\mathrm{CD}^{+} \mathrm{T}$ lymphocyte abundance were significantly higher in lung cancer tissues than in the adjacent normal tissues $(\mathrm{P}<0.01$, Table 2$)$. A negative correlation was observed between the expression levels of GSDME and HMGB1 and lymph node metastasis, ie, patients with lung cancer showing higher expression levels of GSDME and HMGB1 had a lower chance of experiencing lymph node invasion ( $\mathrm{P}<0.01$, Table 3 ). The difference between the lung cancer tissues and the adjacent normal tissues with respect to $\mathrm{CD}^{+} \mathrm{T}$ lymphocyte abundance also had the same significance in relation to lymph node metastasis, ie, patients with higher $\mathrm{CD}^{+} \mathrm{T}$ lymphocyte abundance showed lymph node metastasis to a lesser extent, and the difference was also statistically significant $(\mathrm{P}<0.01$, Table 3$)$.

Kaplan-Meier survival analysis showed that Tumor Node Metastasis (TNM) stage, lymph node invasion, tumor size, and GSDME expression levels were significantly correlated with the overall survival rate of the patients (Figure 2). Unexpectedly, we observed that the expression level of HMGB1 was only negatively correlated with lymph node metastasis, ie, patients with higher HMGB1 expression levels showed lymph node metastases to a lower extent; this difference was statistically significant. However, HMGB1 expression level showed no significant relationship with patient prognosis and TNM stage. Further, to comprehensively analyze the variables that showed statistical significance based on Kaplan-Meier survival analysis, Cox multiple regression analysis was performed to estimate the HR and the 95\% CI. Based on the results thus obtained, GSDME and TNM staging $(\mathrm{p}=0.017$ and $\mathrm{p}=0.026$,

Table 2 Comparison of Protein Expressions of GSDME, HMGBI and Caspase 3 and $\mathrm{CD} 8+\mathrm{T}$ Lymphocyte Abundance in Lung Cancer and Paracancerous Tissues

\begin{tabular}{|l|c|c|c|c|}
\hline \multicolumn{2}{|l|}{} & $\begin{array}{c}\text { Tumor } \\
\text { Tissue } \\
(\mathbf{n}(\%))\end{array}$ & $\begin{array}{c}\text { Peritumor } \\
\text { Tissue } \\
(\mathbf{n}(\%))\end{array}$ & p value \\
\hline GSDME & Low & $58(44.6)$ & $17(13.1)$ & $0.002^{*}$ \\
& High & $52(40)$ & $3(2.3)$ & \\
\hline caspase3 & Low & $17(13.1)$ & $13(10.0)$ & $<0.00 I^{*}$ \\
& High & $93(71.5)$ & $7(5.4)$ & \\
\hline HMGBI & Low & $68(52.3)$ & $18(13.9)$ & $0.002^{*}$ \\
& High & $42(32.3)$ & $2(1.5)$ & \\
\hline CD8 ${ }^{+} T$ lymphocytes & Low & $51(39.2)$ & $17(13.1)$ & $<0.00 I^{*}$ \\
& High & $59(45.4)$ & $3(2.3)$ & \\
\hline
\end{tabular}

Note: *The $p$ value is statistically significant. respectively) were identified as independent prognostic factors for patients with lung cancer (Table 4), while the expression levels of HMGB1 and caspase3, and the abundance of $\mathrm{CD}^{+} \mathrm{T}$ lymphocytes did not show any significant effect on the prognosis and survival of the patients.

To further explore the relationship between the expression levels of GSDME, caspase3, and HMGB1 and the clinical pathological parameters, Spearman's rank correlation analysis was performed. The results obtained showed that the expression level of GSDME not only affected the lymph node metastasis and prognostic survival rate of the patients with lung cancer, but showed a positive correlation between with the expression levels of caspase 3 and HMGB1, as well as $\mathrm{CD}^{+} \mathrm{T}$ lymphocyte abundance. A positive correlation was also observed between the expression level of HMGB1 and the abundance of $\mathrm{CD}^{+} \mathrm{T}$ lymphocytes (correlation coefficient $=$ 0.468 , $\mathrm{P}<0.001$; Table 5). Further, HMGB1 expression level and $\mathrm{CD}^{+} \mathrm{T}$ lymphocyte abundance had no effect on the prognostic survival of the patients. Furthermore, patients with higher HMGB1 expression levels and $\mathrm{CD}^{+} \mathrm{T}$ lymphocyte abundance showed lymph node invasion to a lower extent, and the difference was statistically significant (Table 5).

\section{Discussion}

Cancer (malignant tumor) has become one of the major diseases that threatens human health worldwide, and specifically, the incidence and mortality rate of lung cancer are still high. Further, the primary objective of current treatments for malignant tumors is the induction of tumor cell death or the inhibition of their growth. ${ }^{1}$ This notwithstanding, it is necessary to explore whether the secondary reactions caused by the release of intracellular factors after the death of the tumor cells can promote or inhibit tumors. Therefore, in this study, we explored pyroptosis mediated by caspase3/GSDME in lung cancer tissues. Our resulted indicated that after the destruction of the integrity of the cell membrane and cell death, the passive release of the intracellular factor, HMGB1 (nuclear factor), and the recruitment of $\mathrm{CD}^{+} \mathrm{T}$ lymphocytes had an effect on the clinicopathological parameters of patients with lung cancer as well as their prognostic survival.

Pyroptosis, mediated by the gasdermin protein family, is a programmed cell death mode characterized by a high degree of inflammation, and possibly, it affects various stages of cancer. Therefore, studying its role in cancer progression may highlight new treatment options for cancer. Further, caspase is a cysteine protease that cleaves the 
Table 3 The Relationship Between the Protein Expression of GSDME, HMGBI and Caspase3 and the Abundance of CD8+ T Lymphocytes and the Clinical Parameters of I 10 Lung Cancer Patients

\begin{tabular}{|c|c|c|c|c|c|c|c|c|c|c|c|c|}
\hline \multirow[t]{2}{*}{ Variable } & \multicolumn{3}{|c|}{ GSDME } & \multicolumn{3}{|c|}{ Caspase-3 } & \multicolumn{3}{|c|}{ HMGB I } & \multicolumn{3}{|c|}{$\mathrm{CD8}^{+} \mathrm{T}^{\mathrm{Lymphocytes}}$} \\
\hline & Low & High & P value & Low & High & P value & Low & High & P value & Low & High & P value \\
\hline \multicolumn{13}{|l|}{ Age } \\
\hline$>65$ & 27 & 26 & 0.718 & 8 & 45 & 0.92 & 29 & 24 & 0.139 & 21 & 32 & 0.172 \\
\hline$\leq 65$ & 31 & 26 & & 9 & 48 & & 39 & 18 & & 30 & 27 & \\
\hline \multicolumn{13}{|l|}{ Gender } \\
\hline Male & 47 & 38 & 0.32 & 12 & 73 & 0.474 & 51 & 34 & 0.469 & 39 & 46 & 0.852 \\
\hline Female & 11 & 14 & & 5 & 20 & & 17 & 8 & & 12 & 13 & \\
\hline \multicolumn{13}{|l|}{ Pathologic grade } \\
\hline I & 13 & 14 & 0.752 & 3 & 24 & 0.178 & 18 & 9 & 0.794 & 12 & 15 & 0.824 \\
\hline II & 32 & 25 & & 7 & 50 & & 35 & 22 & & 28 & 29 & \\
\hline III & 1320 & 13 & & 7 & 19 & & 15 & II & & 11 & 15 & \\
\hline \multicolumn{13}{|l|}{ TNM stage24 } \\
\hline I & 13 & 22 & 0.166 & 5 & 30 & 0.897 & 19 & 16 & 0.67 & 14 & 21 & 0.837 \\
\hline II & 20 & 13 & & 6 & 27 & & 21 & 12 & & 16 & 17 & \\
\hline III & 24 & 16 & & 6 & 34 & & 27 & 13 & & 20 & 20 & \\
\hline IV & I & I & & 0 & 2 & & I & I & & I & I & \\
\hline \multicolumn{13}{|c|}{ Lymphatic invasion } \\
\hline Yes(NI,N2,N3) & 31 & 13 & $0.002^{*}$ & 7 & 37 & 0.914 & 33 & 11 & $0.02 *$ & 26 & 18 & $0.029 *$ \\
\hline $\mathrm{No}(\mathrm{N} 0)$ & 27 & 39 & & 10 & 56 & & 35 & 31 & & 25 & 41 & \\
\hline \multicolumn{13}{|l|}{ Vital states } \\
\hline Alive & 15 & 28 & $0.003 *$ & 4 & 39 & 0.153 & 23 & 20 & 0.15 & 17 & 26 & 0.25 \\
\hline Dead & 43 & 24 & & 13 & 54 & & 45 & 22 & & 34 & 33 & \\
\hline \multicolumn{13}{|l|}{ Tumor size } \\
\hline$\leq 5(\mathrm{TI}, \mathrm{T} 2)$ & 44 & 38 & 0.738 & 13 & 69 & 0.843 & 50 & 32 & 0.756 & 40 & 42 & 0.384 \\
\hline$>5(\mathrm{~T} 3, \mathrm{~T} 4)$ & 14 & 14 & & 4 & 24 & & 18 & 10 & & II & 17 & \\
\hline \multicolumn{13}{|c|}{ Expression of GSDME } \\
\hline Low & - & - & - & 14 & 44 & $0.008 *$ & 50 & 8 & $<0.00 I^{*}$ & 40 & 18 & $0.000 *$ \\
\hline High & - & - & & 3 & 49 & & 18 & 34 & & 11 & 41 & \\
\hline \multicolumn{13}{|c|}{ Expression of caspase- 3} \\
\hline Low & 14 & 3 & $0.008^{*}$ & - & - & - & 14 & 3 & 0.058 & 11 & 6 & 0.099 \\
\hline High & 44 & 49 & & - & - & & 54 & 39 & & 40 & 53 & \\
\hline \multicolumn{13}{|c|}{ Expression of HMGBI } \\
\hline Low & 50 & 18 & $<0.001 *$ & 14 & 54 & 0.058 & - & - & - & 44 & 24 & $<0.001 *$ \\
\hline High & 8 & 34 & & 3 & 39 & & - & - & & 7 & 35 & \\
\hline \multicolumn{13}{|c|}{ Abundance of $\mathrm{CD}^{+} \mathrm{T}$ lymphocytes } \\
\hline Low & 40 & II & $0.001 *$ & II & 40 & 0.099 & 44 & 7 & $<0.001 *$ & - & - & - \\
\hline High & 18 & 41 & & 6 & 53 & & 24 & 35 & & - & - & \\
\hline
\end{tabular}

Notes: *The $\mathrm{p}$ value is less than 0.05 , and the difference in protein expression and CD $8+T$ lymphocyte abundance between clinicopathological parameters is statistically significant. 

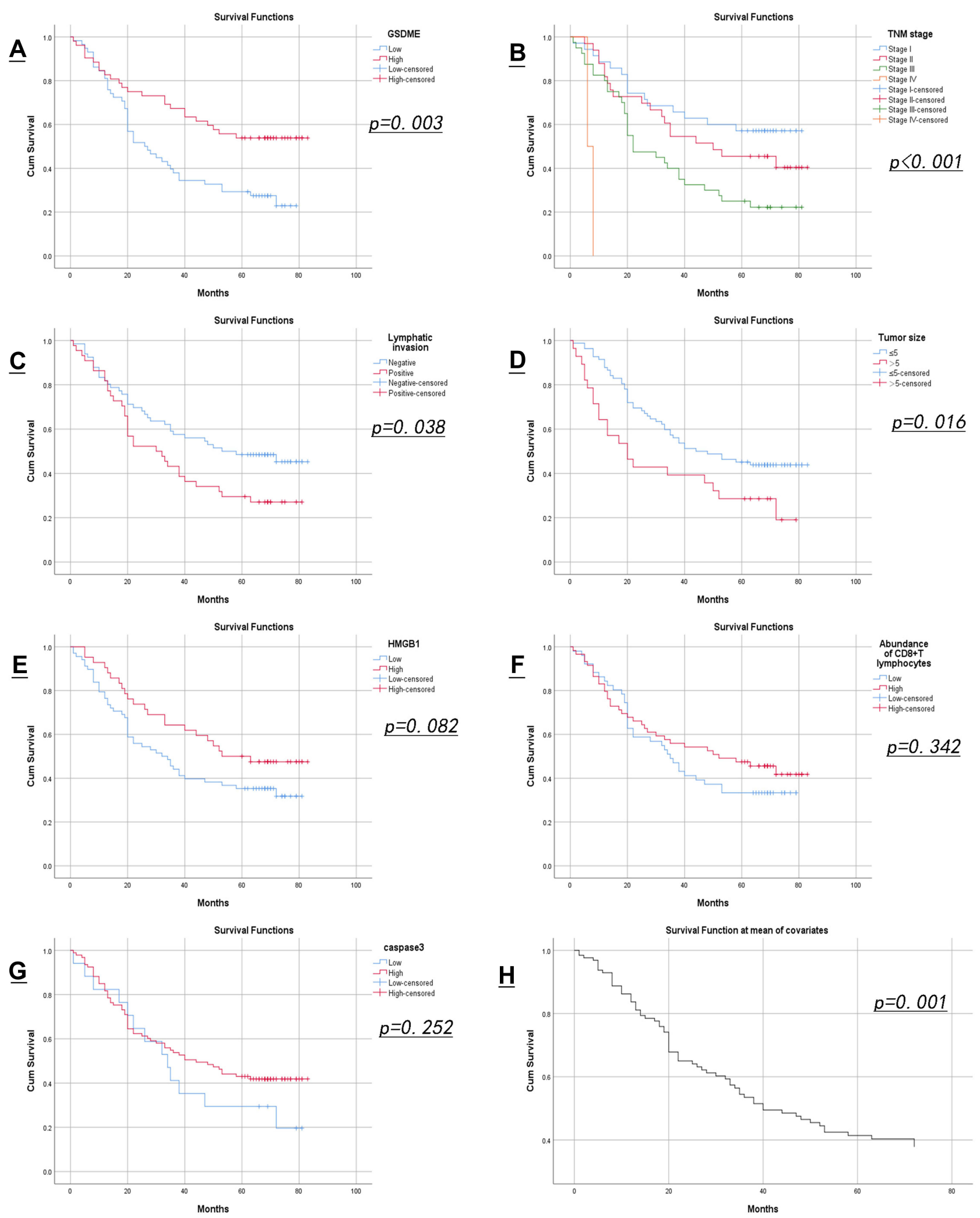

Figure 2 Kaplan-Meier survival curve. In Kaplan-Meier survival analysis, the expression level of GSDME (A), TNM stage (B), lymph node invasion (C) and tumor size (D) were the factors affecting the prognosis of patients $(P=0.003, P=0.000, P=0.016, P=0.038$, respectively). The expression levels of HMGBI (E), caspase3 (G) and the abundance of $C D 8+T$ lymphocytes $(\mathbf{F})$ were not used as prognostic indicators for the survival of lung cancer patients $(P=0.082, P=0.252$, $P=0.342$, respectively). Multivariate analysis of survival curve showed $P=0.001(\mathbf{H})$. In the TNM staging image, lines of different colors represent different TNM staging, as shown in the notes next to the image. Kaplan-Meier survival curve of other variables, red curve represents high expression and blue curve represents low expression. 
Table 4 Univariate and Multivariate Analysis of Overall Survival

\begin{tabular}{|c|c|c|c|c|}
\hline & Univariate Analysis & \multicolumn{3}{|c|}{ Multivariate Analysis } \\
\hline & $\mathrm{P}$ & $P$ & $\mathrm{HR}$ & $95 \% \mathrm{Cl}$ \\
\hline GSDME & $0.004^{*}$ & $0.017 *$ & 0.531 & $0.316-0.893$ \\
\hline caspase-3 & 0.259 & & & \\
\hline HMGBI & 0.088 & & & \\
\hline $\mathrm{CD} 8^{+} \mathrm{T}$ & 0.348 & & & \\
\hline Age & 0.057 & & & \\
\hline Gender & 0.999 & & & \\
\hline TNM stage & $0.001 *$ & $0.026 *$ & 1.65 & $1.06 \mathrm{I}-2.566$ \\
\hline Pathologic grade & 0.178 & & & \\
\hline Lymphatic invasion & $0.042^{*}$ & 0.468 & 0.788 & $0.4|4-1.50|$ \\
\hline Tumor size & $0.019 *$ & 0.456 & 1.25 & $0.695-2.249$ \\
\hline
\end{tabular}

Note: *The $\mathrm{p}$ value is statistically significant.

Abbreviations: $\mathrm{Cl}$, confidence interval; $\mathrm{HR}$, hazard ratio.

Table 5 Correlation Between GSDME, Caspase3, HMGBI and CD8+ T Lymphocytes and Other Clinicopathological Parameters of Lung Cancer Patients

\begin{tabular}{|c|c|c|c|c|c|c|c|c|}
\hline \multirow[t]{2}{*}{ Variables } & \multicolumn{2}{|c|}{ GSDME } & \multicolumn{2}{|c|}{ Caspase 3} & \multicolumn{2}{|c|}{ HMGB I } & \multicolumn{2}{|c|}{$\begin{array}{c}\text { Abundance of CD8 } \\
\text { T Lymphocytes }\end{array}$} \\
\hline & $\rho($ rho $)$ & P-value & $\rho($ rho $)$ & P-value & $\rho($ rho $)$ & P-value & $\rho($ rho $)$ & P-value \\
\hline Age & -0.034 & 0.721 & -0.01 & 0.921 & $-0.14 \mid$ & 0.142 & -0.13 & 0.175 \\
\hline Gender & 0.095 & 0.325 & -0.068 & 0.479 & -0.069 & 0.474 & -0.018 & 0.854 \\
\hline TNM stage & -0.175 & 0.067 & 0.009 & 0.927 & -0.099 & 0.303 & $-0.08 \mathrm{I}$ & 0.401 \\
\hline Pathologic grade & -0.014 & 0.883 & -0.15 & 0.117 & 0.064 & 0.505 & 0.014 & 0.886 \\
\hline Lymphatic invasion & -0.29 & $0.002^{*}$ & -0.01 & 0.915 & -0.222 & $0.020 *$ & -0.208 & $0.029 *$ \\
\hline Vital states & 0.286 & $0.002^{*}$ & 0.136 & 0.156 & 0.137 & 0.152 & 0.11 & 0.254 \\
\hline Tumor size & -0.175 & 0.067 & 0.019 & 0.845 & -0.03 & 0.758 & 0.083 & 0.389 \\
\hline Expression of GSDME & - & - & 0.254 & $0.007^{*}$ & 0.53 & $0.000 *$ & 0.479 & $0.000^{*}$ \\
\hline Expression of caspase- 3 & 0.254 & $0.007^{*}$ & - & - & 0.181 & 0.059 & 0.157 & 0.101 \\
\hline Expression of $\mathrm{HMGBI}$ & 0.53 & $<0.001 *$ & 0.181 & 0.059 & - & - & 0.468 & $<0.001 *$ \\
\hline Abundance of $\mathrm{CD}^{+}{ }^{+} \mathrm{T}$ lymphocytes & 0.479 & $<0.00 I^{*}$ & 0.157 & 0.101 & 0.468 & $<0.00 I^{*}$ & - & - \\
\hline
\end{tabular}

Note: $*$ The $p$ value is statistically significant.

Abbreviation: $\rho$ (rho), Spearman correlation coefficient.

site behind the aspartic acid residue at a specific recognition site, and reportedly, the activation of caspase enzymes is a biochemical marker of apoptosis. ${ }^{14}$ Specifically, caspase 3 is a major effector caspase that is involved in the activation of inflammatory mediators and plays a key role in the apoptotic cascade. ${ }^{14,15}$ However, an increasing number of studies have shown that it has functions that go beyond its previous typically cognized functions. Wang et $\mathrm{al}^{4}$ showed its involvement in the regulation of pyroptosis via the lysing of gasdermin E (GSDME), which when overexpressed, redirects caspase3-mediated apoptosis to pyrolysis.

Consistent with our results, an increasing number of studies have shown that there is a certain level of correlation between caspase 3 and GSDME. The expression levels of GSDME and caspase3 were positively correlated for the 110 cases of lung cancer included in this study. Additionally, it has been reported that activated caspase 3 cleaves GSDME at specific sites to form GSDME-C terminal and GSDME-N terminal domains. ${ }^{16}$ Further, some of the GSDME-N terminal domains become integrated into the lipid components of cell membranes, resulting in pore formation, cytoplasmic swelling, cell rupture, and cell disintegration, as well as the subsequent release of intracellular substances into the extracellular environment, triggering and amplifying secondary inflammatory reactions. ${ }^{17-19}$

HMGB1 is a mobile chromatin binding protein that acts as a DNA molecular chain, and reportedly, its release and 
activation are highly controlled. ${ }^{6}$ Usually, after cell damage or cell death, the cell membrane ruptures and passively releases HMGB1 into the extracellular environment, where it acts as a pro-inflammatory cytokine. ${ }^{7}$ Further, it has been demonstrated that both cytokines and inflammasomes play an important role in tumorigenesis, and may affect cancer pathogenesis by regulating innate and adaptive immune responses. ${ }^{5,20-22}$ Therefore, HMGB1 possibly participates in some internal molecular mechanisms associated with carcinogenesis and could serve as a target for cancer treatment. However, its role in cancer remains controversial. Reportedly, HMGB1 is a highly conserved chromosomal protein in the cell that can act as a DNA molecular chaperone. However, when in the extracellular environment, it is generally regarded as a signaling molecule that is involved in a variety of physical and pathological processes. It also interacts with chemokines and growth factors, among other factors. ${ }^{1,9,23}$ Additionally, extracellular HMGB1 can activate pro-inflammatory signaling pathways, induce the release of pro-inflammatory cytokines, accelerate inflammatory response, and promote tumor formation and metastasis. ${ }^{1}$ As a tumor promoter, HMGB1 released by tumor cells enhances the recruitment of immunosuppressive cells, which in turn promote tumor angiogenesis, invasion, and metastasis. ${ }^{23,24}$ Conversely, Tang et $\mathrm{al}^{25,26}$ and Lange et $\mathrm{al}^{27}$ reported an opposite conclusion, indicating that HMGB1-mediated DNA damage repair, gene recombination, and gene transcription promotion can help maintain the stability of the nucleus. However, it has been observed that HMGB1 loss leads to autophagy defects, which may cause genome instability and tumorigenesis. Conversely, Jiao et $\mathrm{al}^{28}$ also reported that HMGB1 in cells can inhibit breast cancer by directly binding to RB, which is a well-known tumor suppressor protein. This indicates that endogenous HMGB1, as an RB-related protein, inhibits breast tumors. Thus, various reports on the roles and effects of HMGB1 are diametrically opposing, indicating that HMGB1 may play different roles in different cell and tumor types. Therefore, its function and specific role in tumors still need to be further clarified.

Among the 110 patients with lung cancer recruited in this study, 42 (38.18\%) showed high HMGB1 expression levels. Our results also showed the existence of a negative correlation between the expression level of HMGB1 and lymph node metastasis; ie, patients showing high HMGB1 expression levels had less lymph node metastasis. However, no significant relationship was observed between HMGB1 expression and prognostic survival. Therefore, it could not concluded that HMGB1 affects the prognostic survival of patients. Given that patients with high HMGB1 expression levels showed a lower chance of the occurrence of lymph node invasion, we speculated that HMGB1 may be an important factor that inhibits tumor growth and invasion. This implies that enhancing the expression of HMGB1 may be helpful for the treatment of lung cancer.

Our results also indicated that the abundance of $\mathrm{CD} 8^{+}$ $\mathrm{T}$ lymphocytes, with immune surveillance ability on tumor cells in lung cancer tissues, was positively correlated with the expression of GSDME and HMGB1. This finding further confirmed that extracellular HMGB1 is involved in the recruitment of immune cells. Highly differentiated effector $\mathrm{T}$ cells are associated with various disease processes, and the main immune cells, $\mathrm{CD} 8^{+} \mathrm{T}$ lymphocytes, which play a vital role in the occurrence and development of tumors, also play an important role in the anti-tumor process. $^{10,29}$

Zhang et $\mathrm{al}^{11}$ pointed out that GSDME expression can recruit immune cells to the tumor microenvironment and promote their functioning, thereby enhancing anti-tumor adaptive immunity. In turn, $\mathrm{CD} 8{ }^{+} \mathrm{T}$ cells can mediate tumor inhibition by GSDME, thereby establishing a mutually reinforcing function that is conducive to fighting tumor cell immune evasion. In our study, patients with high $\mathrm{CD}^{+} \mathrm{T}$ lymphocyte abundance showed lymph node metastasis to a lesser extent, indicating that $\mathrm{CD} 8^{+}$ $\mathrm{T}$ lymphocytes have the ability to monitor tumor cells and inhibit their metastasis and invasion. Unfortunately, based on our results, it could not be concluded that CD8 ${ }^{+}$ $\mathrm{T}$ lymphocyte abundance is a factor that affects patient prognosis. However, the function of $\mathrm{CD} 8^{+} \mathrm{T}$ lymphocytes in inhibiting tumor metastasis and invasion could not be ignored.

\section{Conclusion}

Overall, in this study, we observed that the expression levels of GSDME, caspase3, and HMGB1 as well as $\mathrm{CD} 8+\mathrm{T}$ lymphocyte abundance were significantly higher in lung cancer tissues than in normal adjacent tissues. Further, based on Cox univariate and multivariate analyses, GSDME and TNM staging were identified as independent factors affecting the prognosis and survival of patients with lung cancer. Even though we could not conclude that the expression level of HMGB1 and CD8+ $\mathrm{T}$ lymphocyte abundance affect the prognosis of patients with lung cancer, their inhibitory effects on tumor cell metastasis and invasion could not be ignored. Therefore, 
further studies regarding their roles in cancer and their specific functional mechanisms are necessary.

\section{Abbreviation}

GSDME, gasdermin E; HMGB1, high mobility group protein; TNM, tumor node metastasis.

\section{Declarations}

We hereby declare that the ethics committee of the First Affiliated Hospital of Bengbu Medical College has approved our informed consent process. In addition, our study was approved by the ethics committee of Bengbu Medical College.[Renke Pi Zi [2021] No. 265]. Finally, due to time- and geographical location-related limitations, oral informed consent was obtained from some of the patients during the follow-up stage before the commencement of the experiments (Please note: To be able to compare the 5-year survival rates of the patients, the study involved cases that were recruited 5-6 years ago). During the study period, some of the patients died, while some changed their home addresses. Therefore, the participation of some patients was via oral explanation during telephone follow-up. Further, consent was obtained from patients or their family members. Some of them provided written informed consent before the start of the experiment.

\section{Data Sharing Statement}

Data sharing is not applicable to this article as no datasets were generated or analyzed during this study.

\section{Ethics Approval and Consent to Participate}

This study, which was conducted in accordance with the ethical guidelines of the Declaration of Helsinki, was approved by the Ethics Committee of the First Affiliated Hospital of Bengbu Medical College.[Renke Pi Zi [2021] No. 265]. Prior to the start of the study, informed consent was obtained from all patients participating in the study.

\section{Consent for Publication}

All authors who participated in this study agreed to publish the paper in your journal.

\section{Acknowledgments}

I would like to thank all the authors for their efforts. In particular, I would like to thank the professor of Pathology Department of the First Affiliated Hospital of Bengbu
Medical College for their support and help to this experiment. Special thanks to "editage" for revising the language of this article.

\section{Funding}

This work was supported by the Graduate Innovation Program of the Bengbu Medical College (grant number Byycx20068).

\section{Disclosure}

The authors declare no conflicts of interest in this work.

\section{References}

1. Kang R, Zhang Q, Zeh HJ 3rd, Lotze MT, Tang D. HMGB1 in cancer: good, bad, or both? Clin Cancer Res. 2013;19 (15):4046-4057. doi:10.1158/1078-0432.CCR-13-0495

2. Islas-Vazquez L, Aguilar-Cazares D, Galicia-Velasco M, et al. IL-6, NLR, and SII markers and their relation with alterations in CD8+ T-lymphocyte subpopulations in patients treated for lung adenocarcinoma. Biology. 2020;9(11):376.

3. Lu T, Yang X, Huang Y, et al. Trends in the incidence, treatment, and survival of patients with lung cancer in the last four decades. Cancer Manag Res. 2019;11:943-953. doi:10.2147/CMAR.S187317

4. Wang Y, Gao W, Shi X, et al. Chemotherapy drugs induce pyroptosis through caspase-3 cleavage of a gasdermin. Nature. 2017;547 (7661):99-103. doi:10.1038/nature22393

5. Hinshaw DC, Shevde LA. The tumor microenvironment innately modulates cancer progression. Cancer Res. 2019;79(18):4557-4566. doi:10.1158/0008-5472.CAN-18-3962

6. Liu A, Yang B. Roles of TRPM7 in renal ischemia-reperfusion injury. Curr Protein Pept Sci. 2019;20(8):777-788. doi:10.2174/ 1389203720666190507102948

7. Simpson J, Loh Z, Ullah MA, et al. Respiratory syncytial virus infection promotes necroptosis and HMGB1 release by airway epithelial cells. Am J Respir Crit Care Med. 2020;201 (11):1358-1371. doi:10.1164/rccm.201906-1149OC

8. Vénéreau E, Ceriotti C, Bianchi ME. DAMPs from cell death to new life. Front Immunol. 2015;6:422. doi:10.3389/fimmu.2015.00422

9. Zhang Z, Wang M, Zhou L, et al. Increased HMGB1 and cleaved caspase-3 stimulate the proliferation of tumor cells and are correlated with the poor prognosis in colorectal cancer. J Exp Clin Cancer Res. 2015;34(1):51. doi:10.1186/s13046-015-0166-1

10. Morris AB, Farley CR, Pinelli DF, et al. Signaling through the inhibitory $\mathrm{Fc}$ receptor Fc $\gamma \mathrm{RIIB}$ induces $\mathrm{CD} 8^{+} \mathrm{T}$ cell apoptosis to limit $\mathrm{T}$ cell immunity. Immunity. 2020;52(1):136-150.e6. doi:10.1016/j.immuni.2019.12.006

11. Zhang Z, Zhang Y, Xia S, et al. Gasdermin E suppresses tumour growth by activating anti-tumour immunity. Nature. 2020;579 (7799):415-420. doi:10.1038/s41586-020-2071-9

12. Wu M, Wang Y, Yang D, et al. A PLK1 kinase inhibitor enhances the chemosensitivity of cisplatin by inducing pyroptosis in oesophageal squamous cell carcinoma. EbioMedicine. 2019;41:244-255. doi:10.1016/j.ebiom.2019.02.012

13. Liu Z, Zhou Q, Wang Z, et al. Intratumoral $\mathrm{TIGIT}^{+} \mathrm{CD}^{+}{ }^{+}$-cell infiltration determines poor prognosis and immune evasion in patients with muscle-invasive bladder cancer. J Immunother Cancer. 2020;8 (2):e000978. doi:10.1136/jitc-2020-000978

14. Yang S, Thor AD, Edgerton S, Yang X. caspase-3 mediated feedback activation of apical caspases in doxorubicin and TNF-alpha induced apoptosis. Apoptosis. 2006;11(11):1987-1997. doi:10.1007/s10495006-0084-y 
15. Porter AG, Jänicke RU. Emerging roles of caspase-3 in apoptosis. Cell Death Differ. 1999;6(2):99-104. doi:10.1038/sj.cdd.4400476

16. Wang YY, Liu XL, Zhao R. Induction of pyroptosis and its implications in cancer management. Front Oncol. 2019;9:971. doi:10.3389/ fonc. 2019.00971

17. Fink SL, Cookson BT. Pyroptosis and host cell death responses during salmonella infection. Cell Microbiol. 2007;9(11):2562-2570. doi:10.1111/j.1462-5822.2007.01036.x

18. Ding J, Wang K, Liu W, et al. Pore-forming activity and structural autoinhibition of the gasdermin family [publIshed correctIon appears In Nature. Nature. 2016;535(7610):111-116. doi:10.1038/ nature 18590

19. Aglietti RA, Dueber EC. Recent insights into the molecular mechanisms underlying pyroptosis and gasdermin family functions. Trends Immunol. 2017;38(4):261-271. doi:10.1016/j.it.2017.01.003

20. Moossavi M, Parsamanesh N, Bahrami A, Atkin SL, Sahebkar A. Role of the NLRP3 inflammasome in cancer. Mol Cancer. 2018;17 (1):158. doi:10.1186/s12943-018-0900-3

21. Dunn JH, Ellis LZ, Fujita M. Inflammasomes as molecular mediators of inflammation and cancer: potential role in melanoma. Cancer Lett. 2012;314(1):24-33. doi:10.1016/j.canlet.2011.10.001

22. Karki R, Man SM, Kanneganti TD. Inflammasomes and cancer. Cancer Immunol Res. 2017;5(2):94-99. doi:10.1158/2326-6066. CIR-16-0269
23. Sims GP, Rowe DC, Rietdijk ST, Herbst R, Coyle AJ. HMGB1 and RAGE in inflammation and cancer. Annu Rev Immunol. 2010;28:367-388. doi:10.1146/annurev.immunol.021908.132603

24. Gao Q, Wang S, Chen X, et al. Cancer-cell-secreted CXCL11 promoted $\mathrm{CD} 8^{+} \mathrm{T}$ cells infiltration through docetaxel-induced-release of HMGB1 in NSCLC. $J$ Immunother Cancer. 2019;7(1):42. doi:10.1186/s40425-019-0511-6

25. Tang D, Kang R, Livesey KM, et al. Endogenous HMGB1 regulates autophagy. J Cell Biol. 2010;190(5):881-892. doi:10.1083/ jcb.200911078

26. Tang D, Kang R, Livesey KM, et al. High-mobility group box 1 is essential for mitochondrial quality control. Cell Metab. 2011;13 (6):701-711. doi:10.1016/j.cmet.2011.04.008

27. Lange SS, Vasquez KM. HMGB1: the jack-of-all-trades protein is a master DNA repair mechanic. Mol Carcinog. 2009;48(7):571-580. doi:10.1002/mc.20544

28. Jiao Y, Wang HC, Fan SJ. Growth suppression and radiosensitivity increase by HMGB1 in breast cancer. Acta Pharmacol Sin. 2007;28 (12):1957-1967. doi:10.1111/j.1745-7254.2007.00669.x

29. Vesely MD, Kershaw MH, Schreiber RD, et al. Natural innate and adaptive immunity to cancer. Annu Rev Immunol. 2011;29:235-271. doi:10.1146/annurev-immunol-031210-101324
Cancer Management and Research

\section{Publish your work in this journal}

Cancer Management and Research is an international, peer-reviewed open access journal focusing on cancer research and the optimal use of preventative and integrated treatment interventions to achieve improved outcomes, enhanced survival and quality of life for the cancer patient.
Dovepress

The manuscript management system is completely online and includes a very quick and fair peer-review system, which is all easy to use. Visit http://www.dovepress.com/testimonials.php to read real quotes from published authors. 